\title{
Effect of Crotonylidene Di-Urea on Yield and Quality of Banana on Inceptisol
}

\author{
Atul Pawar*, M.R. Chauhan and Pramod Gawade
}

\author{
Department of Soil Science and Agricultural Chemistry, Mahatma Phule Krishi Vidyapeeth, \\ Rahuri, Ahmednagar, Maharashtra, India \\ *Corresponding author
}

\begin{tabular}{|c|c|}
\hline & A B S T R A C T \\
\hline & $\begin{array}{l}\text { Field experiment was conducted at the research farm of Soil Test Crop Response } \\
\text { (STCR) Correlation Project, Mahatma PhuleKrishi Vidyapeeth, Rahuri during 2015- }\end{array}$ \\
\hline Keywords & $\begin{array}{l}16 \text { to study the effect of crotonylidenediurea on yield and quality of banana on } \\
\text { inceptisol. The growth parameters of banana, number of leaves and pseudostem girth }\end{array}$ \\
\hline $\begin{array}{l}\text { Crotonylidene } \\
\text { diurea, Nitrogen, } \\
\text { Yield and } \\
\text { quality of banana }\end{array}$ & $\begin{array}{l}\text { was on par with each other in treatment GRDN and } 100 \% \mathrm{~N} \text { through CDU. The yield } \\
\text { attributing characters, number hands, number of fingers per bunch and bunch weight } \\
\text { was on par in treatment GRDN and } 100 \% \mathrm{~N} \text { through CDU. The banana yield and } \\
\text { quality viz., total sugar, reducing sugars and pulp: peel ratio was significantly higher }\end{array}$ \\
\hline Article Info & $\begin{array}{l}\text { in treatment GRDN and statistically on par with } 100 \% \mathrm{~N} \text { through CDU. Thus, the } \\
\text { addition of nitrogen through urea and crotonylidenediurea as per general }\end{array}$ \\
\hline $\begin{array}{l}\text { Accepted: } \\
19 \text { April } 2017 \\
\text { Available Online: } \\
10 \text { May } 2017\end{array}$ & $\begin{array}{l}\text { recommended dose of nutrients in an incubation study was found beneficial for } \\
\text { periodical soil enzyme activity, microbial population, ammonical and nitrate nitrogen } \\
\text { and soil available phosphorus and potassium. The banana yield and banana yield } \\
\text { attributing character and residual soil fertility was benefited by an application of }\end{array}$ \\
\hline & $\begin{array}{l}\text { nitrogen through urea and } 100 \% \text { through CDU as GRDN. The GRDN are beneficial } \\
\text { for nitrogen, phosphorus and potassium uptake }\left(582.27 \mathrm{~kg} \mathrm{ha}^{-1}, 156.27 \mathrm{~kg} \mathrm{ha}^{-1} \text { and }\right. \\
1031.7 \mathrm{~kg} \mathrm{ha}^{-1} \text { respectively) }\end{array}$ \\
\hline
\end{tabular}

\section{Introduction}

The banana is botanically, Musa Sp. also referred to as the tree of paradise. India, with an annual production of 26.22 million tonnes from 4,04,000, ha area is the largest producer of banana in the world. It contributes $27 \%$ of the global production and about $37 \%$ of total fruit crop production in the country (FAO 2015). Grand Naine is a popular variety of banana grown mostly in all export oriented countries of Asia, South America and Africa due to its excellent fruit quality.
This is a superior selection of Giant Cavendish introduced to India in 1990's (Singh and Chundawat, 2002). Among the Indian States, Maharashtra contributes maximum area of about 90,000 ha for banana cultivation and accounts for $25 \%$ of the total banana production. Despite being the most productive, contribution of banana accounts only $0.1 \%$ to world trade (Aquil et al., 2012).

Nitrogen is essential and primary nutrient, required by all the crops in large amount. 
However, nitrogen fertilizer added in soil get leached or washed out. It not only causes economic loss but also gives invitation to soil, water and environmental pollution. It has harmful effect on soil health as well as human health, the use of slow release fertilizer up to some extent useful and ecofriendly option to overcome these problems. Excess nitrogen in banana promotes pseudosteme longation which results in plant lodging and consequently yield loss, an oversupply of nitrogen increases the time needed for banana fruit filling and affects fruit quality (Lahav, 1995). Excessive nitrogen fertilization also increases the nutrient loss into the environment by leaching, denitrification and volatilization (Follett, 2001). These losses have the potential of disrupting the environment. To ameliorate this problem, recommended that nitrogen fertilization should be kept to the lowest level that is consistent with an optimum yield (Lorenz, 1978). Urea is the widely used nitrogenous fertilizer in agriculture because of its high nitrogen content (46\%). However, 50-70 per cent of the applied nitrogen lost due to different losses via volatilization and leaching reducing the use efficiency of applied fertilizers (Shaviv and Mikkelsen, 1993). This reduces the productivity and increases the cost of cultivation besides polluting the environment. Increasing the nitrogen use efficiency will lead to increase in productivity \& substantially. Various methods recommended to increase fertilizer use efficiency are increasing the organic matter content of soil through application of organic manures, split application of fertilizers and application of coated or slow release fertilizers. In this context, the controlled release technology by coating urea with different materials such as phosphogypsum, sulphur, resin polymers, Dicyclo-pentadiene (DCPD), pine tree Kraft lignin and neem using different techniques of rotating drum, fluidized bed and spouted bed to increase the efficiency of urea fertilizer has been investigated (Susherman and Anggoro, 2011). It was reported that the thickness of coating fertilizers, affects the release pattern of nitrogen from fertilizers.

Studies of enzyme activities in soil are important as they indicate the potential of the soil to support biochemical processes which are essential for the maintenance of soil fertility (Dkhar and Mishra, 1983). Any management practice that influences microbial communities in soil may be expected to produce changes in soil enzyme activity level (Perucci et al., 1984). Soil dehydrogenase activity is often used as a measure of any disruption caused by pesticides, trace elements or management (Reddy and Faza, 1989, Wilke, 1991, Frank and Malkomes, 1993). In view of the above considerations, the present research work is planned and conducted at Soil Test Crop Responses Correlation and Project Research Farm, M.P.K.V., Rahuri.

\section{Materials and Methods}

The present investigation was carried out by conducting a field experiment entitled, "Effect of Crotonylidene Diurea on Yield and Quality of Banana on Inceptisol" at Soil Test Crop Response Correlation Project (ICAR), Research Farm, Mahatma PhuleKrishi Vidyapeeth, Rahuri during 2014-16. Geographically the central campus of Mahatma PhuleKrishi Vidyapeeth, Rahuri is situated $38 \mathrm{~km}$ away from Ahmednagar, on Ahmednagar-Manmad State Highway. It lies between 190 48' $\mathrm{N}$ and 190 57' N latitude and 740 19' E longitude. The altitude varies from 495 to 569 meters above mean sea level. This tract is lying on the Eastern side of Western ghat and falls under rain shadow area. The soils of the experimental site of the present investigation was grouped under inceptisols order belong to Sawargaon (Pather) soil series 
which comprises of fine montmorillioniticiso hyperthemic family of VerticHaplustepts. The soil was medium black with $80 \mathrm{~cm}$ depth having swell shrink property. The texture of the soil was clayey with low in available nitrogen (188.16 kg ha-1), medium in available phosphorus (21.43 kg ha-1) and very high in potassium (548.80 $\mathrm{kg}$ ha-1). The soil was slightly alkaline in reaction $(\mathrm{pH} 8.12)$ with calcium carbonate content of $7.90 \%$. Urease activity was $19.60 \mathrm{ug} \mathrm{NH} 4-\mathrm{N}$ g-1 of soil hr-1 and Dehydrogenase activity was 0.97 ug TPF g-1 soil hr-1. Banana crop (Grand Naine) was taken as a test crop in the present investigation, the fruit, shoot \& leaf yield of banana were recorded at harvest. The treatment wise representative fruit, shoot and leaf samples were collected from each plot for studied the uptake of NPK. The representative five plants were tagged for observations from each treatment replication wise. These plants were used to record the number of leaves, height, pseudostem girth, bunch weight, number of hands per bunch, banana yield, pseudostem yield, dry matter yield and nutrient concentration in banana fruits and leaves. Treatment wise soil samples were collected from all the replications at grand growth, shooting and at harvest stage and analysed for residual soil fertility by adopting standard analytical procedure. The soil enzyme activity viz., urease and dehydrogenase were also reported.

Experiment was laid out in randomized block design with four replications comprising of six treatment viz., general recommended dose of nutrients (GRDN) and nitrogen @ $100 \%$, $75 \%, \quad 50 \%, \quad 25 \%$ through crotonylidenediurea (CDU), and absolute control. The FYM was applied @ $10 \mathrm{~kg}$ plantlexcept absolute control treatment. Tissue cultured plantlets of banana (Musa sp.cv Grand Naine) were planted at $1.75 \mathrm{~m} \mathrm{x}$ $1.75 \mathrm{~m}$ spacing. Nitrogen was applied in seven splits, phosphorus was applied at the time of planting, potassium was applied in four splits and FYM at the time of planting. Representative soil samples were collected from each plot at $20 \mathrm{~cm}$ depth before planting of banana, at juvenile, grand growth, shooting and at harvest stage. In standing crop soil sample was taken from the point in between two water drippers and within the active root zone area. The collected samples were air dried in shade on paper sheet, gently ground, mixed and sieved through $2 \mathrm{~mm}$ sieve. The processed soil samples were used for analysis of chemical properties by adopting standard methods of analysis. Total fresh weights of plants from each plot were recorded. The leaves and pseudostem were partitioned in equal parts and a representative composite sample was drawn. The middle three fruits of third hand from the top were selected and cut into small slices. The collected samples were air dried followed by oven dried at $70^{\circ} \mathrm{C}$ temperature till constant weight. Dry matter yield was recorded. The uptake of major nutrients was determined by using dry matter present and nutrient concentration in different plant parts at harvest of banana.

\section{Results and Discussion}

The growth parameters viz., pseudostem height, pseudostem girth, number of functional leaves, are presented in Table 1.The pseduostem height was significantly more in GRDN over the rest of treatment. The nitrogen application to banana through GRDN numerically recorded higher pseudostem height $(261.30 \mathrm{~cm})$ followed by $100 \%$ $(256.65 \mathrm{~cm})$ and $50 \% \mathrm{~N}$ through CDU $(246.10 \mathrm{~cm})$ respectively. Similar results reported by Allen (1986), Alvan and Tucker (1996), Ram (1999) and Badgujar et al., (2004). The pseudostem girth was significantly influenced by the application of CDU (Table 1). The pseudostem girth was significantly more in GRDN $(63.05 \mathrm{~cm})$ than rest of treatment and statistically on par with 
$100 \% \mathrm{~N}$ through CDU(62.75 cm). The pseudostem girth of banana was statistically on par with each other for 75 and $50 \%$ nitrogen application through CDU(61.50 and $60.95 \mathrm{~cm}$ ), the absolute control treatment having the minimum pseudostem girth compared to all treatment. These results are accordance with those reported by Babu et al., (2004). The number of leaves and was significantly more in GRDN (12.45) than rest of treatment and statistically on par with 100 $\% \mathrm{~N}$ through CDU (12.35).

Application of CDU exerted positive influence on yield and yield attributing characters viz., number of hands per bunch, number of fingers per bunch, and bunch weight (Table 2). The number of hands per bunch was significantly higher and statistically on par with each other in treatment GRDN, (12.25) and $100 \% \mathrm{~N}$ through CDU (12.20). The number of hands per bunch was significantly higher and statistically on par with each other in treatment GRDN, (197.50) and $100 \% \mathrm{~N}$ through CDU (195.20). However, it was numerically decreased in 75, 50 and $25 \% \mathrm{~N}$ through CDU (Table 2). The bunch weight and was significantly higher in GRDN (22.24 $\mathrm{kg}$ ) and $100 \% \mathrm{~N}$ through CDU (21.58) over rest of the treatment but statistically on par with each other. However, $75 \% \mathrm{~N}$ and $50 \% \mathrm{~N}$ through CDU to banana recorded statistically on par bunch weight (20.86 and $19.97 \mathrm{~kg}$ ). Similar trend of results were also reported by Mustaffa (1988) and Srinivas et al., (2001). The data on yield are presented in Table 3, revealed that the application of GRDN recorded the highest banana yield (72.61 tha1) and $100 \% \mathrm{~N}$ through $\mathrm{CDU}(70.45 \mathrm{t}$ ha-1) over rest of the treatment but statistically on par with each other. This increase was also associated with the corresponding increase in the number of hands per bunch, number of fingers per bunch and higher bunch weight. However, $75 \% \mathrm{~N}$ and $50 \% \mathrm{~N}$ through
CDUto banana recorded statistically on par (68.12 and $65.21 \mathrm{t}$ ha-1) to banana yield. The increased banana yield and yield attributing characters with increase in nitrogen level was largely due to the increase in morphological traits and also due to higher nutrient uptake by the plants. Significantly lower banana yield $55.66 \mathrm{t}$ ha-1 was recorded in the treatment where no fertilizers and FYM was applied. Similar finding have been also reported by Gour et al., (1990), Mansour et al., (2007) and Kandil et al., (2010). The total $\mathrm{N}, \mathrm{P}$ and $\mathrm{K}$ uptake by banana are presented in Table 3.

The GRDN was recorded significantly higher nitrogen uptake $(582.27 \mathrm{~kg}$ ha-1) than the other treatment but at par with $100 \%$ nitrogen through CDU (545.67 kg ha-1). The treatment of $75 \%, 50 \%$ and $25 \% \mathrm{~N}$ through CDU recorded $489.79,404.48$ and $345.02 \mathrm{~kg}$ ha-1 nitrogen uptake respectively. The significantly higher phosphorus uptake of $156.27 \mathrm{~kg}$ ha-1 was recorded by treatment GRDN, which was at par with $100 \% \mathrm{~N}$ through CDU (144.36). The treatment of T3, T4, T5, recorded phosphorus uptake of 120.06, 105.11 and $80.32 \mathrm{~kg}$ ha-1, respectively. Control treatment recorded $66.79 \mathrm{~kg}$ ha-1 phosphorus uptake (Table 3). The total potassium uptake was significantly more in GRDN (1031.7 kg ha-1) than rest of treatment and statistically on par with $100 \% \mathrm{~N}$ through CDU(998.9 kg ha-1). The uptake of potassium was higher than nitrogen and phosphorus and the sequence of uptake was $\mathrm{K}>\mathrm{N}>\mathrm{P}$. Similar trend of results are also reported by Srinivas et al., (2001). The quality parameters of banana after ripening viz., reducing sugars, non-reducing gars, total sugars, pulp: peel ratio of banana fruit are presented in Table 4 . The total sugars and non reducing sugars content was significantly more in GRDN (17.22 and 2.12\%) and statistically on par with $100 \% \mathrm{~N}$ through CDU(16.98 and 2.07\%). 
Table.1 Effect of crotonylidenedi urea on growth parameters of banana

\begin{tabular}{|c|c|c|c|c|}
\hline $\begin{array}{l}\text { Sr. } \\
\text { No. }\end{array}$ & Treatment & $\begin{array}{l}\text { No. of } \\
\text { leaves }\end{array}$ & $\begin{array}{c}\text { Pseudostem girth } \\
\text { (cm) }\end{array}$ & $\begin{array}{l}\text { Pseudostem } \\
\text { height }(\mathrm{cm})\end{array}$ \\
\hline 1. & $\begin{array}{l}\text { GRDN (200:40:200 g N:P } \mathrm{P}_{2} \mathrm{O}_{5}: \\
\left.\mathrm{K}_{2} \mathrm{O}+10 \mathrm{~kg} \mathrm{FYM} \mathrm{plant}^{-1}\right)\end{array}$ & 12.45 & 63.05 & 261.30 \\
\hline 2. & $\begin{array}{l}100 \% \text { N-CDU+ }\left(40: 200 \text { g }_{2} \mathrm{O}_{5}:\right. \\
\left.\mathrm{K}_{2} \mathrm{O}+10 \mathrm{~kg} \mathrm{FYM} \mathrm{plant}^{-1}\right)\end{array}$ & 12.35 & 62.75 & 256.65 \\
\hline 3. & $\begin{array}{l}75 \% \text { N- CDU+ }\left(40: 200 \text { g }_{2} \mathrm{O}_{5}:\right. \\
\left.\mathrm{K}_{2} \mathrm{O}+10 \mathrm{~kg} \mathrm{FYM} \mathrm{plant}{ }^{-1}\right)\end{array}$ & 11.65 & 61.50 & 251.95 \\
\hline 4. & $\begin{array}{l}50 \% \text { N-CDU+ }\left(40: 200 \text { g }_{2} \mathrm{O}_{5}:\right. \\
\left.\mathrm{K}_{2} \mathrm{O}+10 \mathrm{~kg} \mathrm{FYM} \mathrm{plant}^{-1}\right)\end{array}$ & 11.05 & 60.95 & 246.10 \\
\hline 5 . & $\begin{array}{l}25 \% \text { N- CDU+ }\left(40: 200 \text { g }_{2} \mathrm{O}_{5}:\right. \\
\left.\mathrm{K}_{2} \mathrm{O}+10 \mathrm{~kg} \mathrm{FYM} \mathrm{plant}{ }^{-1}\right)\end{array}$ & 10.95 & 59.10 & 240.45 \\
\hline \multirow[t]{4}{*}{6.} & Absolute control & 10.75 & 58.35 & 236.30 \\
\hline & S.E. \pm & 0.194 & 0.432 & 0.651 \\
\hline & $\mathrm{CD}$ at $5 \%$ & 0.585 & 1.303 & 1.962 \\
\hline & Mean & 11.53 & 60.95 & 248.79 \\
\hline
\end{tabular}

Table. 2 Effect of crotonylidenedi urea on yield attributing characters of banana

\begin{tabular}{|c|c|c|c|c|}
\hline $\begin{array}{l}\text { Sr. } \\
\text { No. }\end{array}$ & Treatment & $\begin{array}{l}\text { No. of hands } \\
\text { bunch }^{-1}\end{array}$ & $\begin{array}{l}\text { No. of fingers } \\
\text { bunch }^{-1}\end{array}$ & $\begin{array}{l}\text { Bunch wt. } \\
(\text { kg) }\end{array}$ \\
\hline 1. & $\begin{array}{l}\text { GRDN (200:40:200 g N:P } \mathrm{O}_{5}: \mathrm{K}_{2} \mathrm{O} \\
\left.+10 \mathrm{~kg} \text { FYM plant }{ }^{-1}\right)\end{array}$ & 12.25 & 197.50 & 22.24 \\
\hline 2. & $\begin{array}{l}100 \% \text { N-CDU+ }\left(40: 200 \text { g P }_{2} \mathrm{O}_{5}\right. \\
\left.\mathrm{K}_{2} \mathrm{O}+10 \mathrm{~kg} \mathrm{FYM} \mathrm{plant}^{-1}\right)\end{array}$ & 12.20 & 195.20 & 21.58 \\
\hline 3. & $\begin{array}{l}75 \% \text { N- CDU+ }\left(40: 200 \text { g }_{2} \mathrm{O}_{5}:\right. \\
\left.\mathrm{K}_{2} \mathrm{O}+10 \mathrm{~kg} \mathrm{FYM} \mathrm{plant}^{-1}\right)\end{array}$ & 11.25 & 185.75 & 20.86 \\
\hline 4. & $\begin{array}{l}50 \% \text { N-CDU+ }\left(40: 200 \text { g }_{2} \mathrm{O}_{5}:\right. \\
\left.\mathrm{K}_{2} \mathrm{O}+10 \mathrm{~kg} \mathrm{FYM} \mathrm{plant}^{-1}\right)\end{array}$ & 10.80 & 180.03 & 19.97 \\
\hline 5. & $\begin{array}{l}25 \% \text { N- CDU+ }\left(40: 200 \text { g }_{2} \mathrm{O}_{5}:\right. \\
\left.\mathrm{K}_{2} \mathrm{O}+10 \mathrm{~kg} \mathrm{FYM} \mathrm{plant}{ }^{-1}\right)\end{array}$ & 10.40 & 172.80 & 18.26 \\
\hline 6. & Absolute control & 09.40 & 165.95 & 17.05 \\
\hline & S.E. \pm & 0.136 & 1.231 & 0.370 \\
\hline & $\mathrm{CD}$ at $5 \%$ & 0.411 & 3.712 & 1.116 \\
\hline & Mean & 11.05 & 182.87 & 19.99 \\
\hline
\end{tabular}


Table.3 Effect of crotonylidenedi urea on yield and nutrient uptake of banana

\begin{tabular}{|c|c|c|c|c|c|c|c|}
\hline \multirow{2}{*}{ Sr.no. } & \multirow{2}{*}{ Treatment } & \multicolumn{3}{|c|}{$\begin{array}{c}\text { Yield } \\
\left(\text { MT/ha }^{-1}\right)\end{array}$} & \multicolumn{3}{|c|}{$\begin{array}{l}\text { Total Nutrient uptake } \\
\qquad\left(\mathrm{kg} \mathrm{ha}^{-1}\right)\end{array}$} \\
\hline & & Fruit & Shoot & Leaves & $\mathbf{N}$ & $\mathbf{P}$ & $\mathbf{K}$ \\
\hline 1 & $\begin{array}{l}\text { GRDN }\left(200: 40: 200{\mathrm{~g} \mathrm{~N}: \mathrm{P}_{2} \mathrm{O}_{5}:}\right. \\
\left.\mathrm{K}_{2} \mathrm{O}+10 \mathrm{~kg} \mathrm{FYM} \mathrm{plant}^{-1}\right)\end{array}$ & 72.60 & 122.67 & 29.47 & 582.27 & 156.27 & 1031.7 \\
\hline 2 & $\begin{array}{l}100 \% \text { N-CDU+ }\left(40: 200 \text { g P}_{2} \mathrm{O}_{5}\right. \\
\left.\mathrm{K}_{2} \mathrm{O}+10 \mathrm{~kg} \mathrm{FYM} \mathrm{plant}^{-1}\right)\end{array}$ & 70.44 & 117.75 & 27.58 & 545.67 & 144.36 & 998.59 \\
\hline 3 & $\begin{array}{l}75 \% \text { N- CDU+ }\left(40: 200 \text { g }_{2} \mathrm{O}_{5}:\right. \\
\left.\mathrm{K}_{2} \mathrm{O}+10 \mathrm{~kg} \mathrm{FYM} \mathrm{plant}^{-1}\right)\end{array}$ & 68.12 & 113.17 & 25.56 & 489.79 & 120.06 & 908.93 \\
\hline 4 & $\begin{array}{l}50 \% \text { N-CDU+ }\left(40: 200 \mathrm{~g} \mathrm{P}_{2} \mathrm{O}_{5}:\right. \\
\left.\mathrm{K}_{2} \mathrm{O}+10 \mathrm{~kg} \mathrm{FYM} \mathrm{plant}^{-1}\right)\end{array}$ & 65.21 & 107.22 & 22.52 & 404.48 & 105.11 & 803.12 \\
\hline 5 & $\begin{array}{l}25 \% \mathrm{~N}-\mathrm{CDU}+\left(40: 200 \mathrm{~g} \mathrm{P}_{2} \mathrm{O}_{5}:\right. \\
\left.\mathrm{K}_{2} \mathrm{O}+10 \mathrm{~kg} \mathrm{FYM} \mathrm{plant}{ }^{-1}\right)\end{array}$ & 59.62 & 101.68 & 20.56 & 345.02 & 80.32 & 746.26 \\
\hline \multirow[t]{3}{*}{6} & Absolute control & 55.65 & 97.59 & 19.36 & 286.83 & 66.79 & 638.50 \\
\hline & S.E. \pm & 1.209 & 1.548 & 0.616 & 16.903 & 4.00 & 18.010 \\
\hline & $\mathrm{CD}$ at $5 \%$ & 3.645 & 4.667 & 1.858 & 50.952 & 12.07 & 54.289 \\
\hline
\end{tabular}

Table.4 Effect of crotonylidenedi urea on quality parameters of banana

\begin{tabular}{|c|c|c|c|c|c|}
\hline $\begin{array}{l}\text { Sr. } \\
\text { No. }\end{array}$ & Treatment & $\begin{array}{c}\text { Total } \\
\text { sugars }(\%)\end{array}$ & $\begin{array}{c}\text { Reducing } \\
\text { sugars } \\
(\%)\end{array}$ & $\begin{array}{c}\text { Non } \\
\text { reducing } \\
\text { sugars }(\%)\end{array}$ & $\begin{array}{c}\text { Pulp:peel } \\
\text { ratio }\end{array}$ \\
\hline 1. & 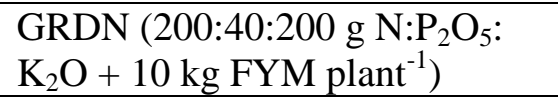 & 17.27 & 15.04 & 2.12 & 3.60 \\
\hline 2. & $\begin{array}{l}100 \% \mathrm{~N}-\mathrm{CDU}+\left(40: 200 \mathrm{~g} \mathrm{P}_{2} \mathrm{O}_{5}:\right. \\
\mathrm{K}_{2} \mathrm{O}+10 \mathrm{~kg} \text { FYM plant } \\
\end{array}$ & 16.98 & 14.79 & 2.07 & 3.48 \\
\hline 3. & $\begin{array}{l}75 \% \text { N- CDU+ }\left(40: 200 \text { g P }_{2} \mathrm{O}_{5}:\right. \\
\mathrm{K}_{2} \mathrm{O}+10 \mathrm{~kg} \mathrm{FYM} \mathrm{plant}\end{array}$ & 16.75 & 14.70 & 1.95 & 3.37 \\
\hline 4. & $\begin{array}{l}50 \% \text { N-CDU+ }\left(40: 200{\text { g } \mathrm{P}_{2} \mathrm{O}_{5}}\right. \\
\left.\mathrm{K}_{2} \mathrm{O}+10 \mathrm{~kg} \mathrm{FYM} \mathrm{plant}^{-1}\right)\end{array}$ & 16.41 & 14.66 & 1.66 & 3.20 \\
\hline 5. & $\begin{array}{l}25 \% \text { N- CDU+ }\left(40: 200 \text { g }_{2} \mathrm{O}_{5}:\right. \\
\left.\mathrm{K}_{2} \mathrm{O}+10 \mathrm{~kg} \mathrm{FYM} \mathrm{plant}{ }^{-1}\right)\end{array}$ & 16.06 & 14.20 & 1.77 & 3.12 \\
\hline \multirow[t]{3}{*}{6.} & Absolute control & 15.71 & 14.16 & 1.48 & 2.90 \\
\hline & S.E. \pm & 0.128 & 0.141 & 0.075 & 0.036 \\
\hline & $\mathrm{CD}$ at $5 \%$ & 0.386 & 0.427 & 0.226 & 0.110 \\
\hline
\end{tabular}


Table.5 Effect of crotonylidenedi urea on soil available nutrients

\begin{tabular}{|c|c|c|c|c|c|c|c|c|c|c|}
\hline \multirow{3}{*}{$\begin{array}{l}\text { Sr. } \\
\text { No }\end{array}$} & \multirow{3}{*}{ Treatment } & \multicolumn{9}{|c|}{ Soilavailable NPK $\left(\mathrm{kg} \mathrm{ha}^{-1}\right)$} \\
\hline & & \multicolumn{3}{|c|}{ Grand growth } & \multicolumn{3}{|c|}{ Shooting stage } & \multicolumn{3}{|c|}{ Harvest stage } \\
\hline & & $\mathbf{N}$ & $\mathbf{P}$ & $\mathbf{K}$ & $\mathbf{N}$ & $\mathbf{P}$ & $\mathbf{K}$ & $\mathbf{N}$ & $\mathbf{P}$ & $\mathbf{K}$ \\
\hline 1. & $\begin{array}{l}\text { GRDN }(200: 40: 200 \mathrm{~g} \\
\mathrm{N}: \mathrm{P}_{2} \mathrm{O}_{5}: \mathrm{K}_{2} \mathrm{O}+10 \mathrm{~kg} \\
\left.\text { FYM plant }^{-1}\right)\end{array}$ & 236.77 & 23.22 & 546.00 & 206.98 & 17.03 & 504.00 & 178.75 & 14.54 & 478.8 \\
\hline 2. & $\begin{array}{l}100 \% \mathrm{~N}-\mathrm{CDU}+(40: 200 \\
\mathrm{g} \mathrm{P}_{2} \mathrm{O}_{5}: \mathrm{K}_{2} \mathrm{O}+10 \mathrm{~kg} \\
\left.\mathrm{FYM}_{\text {plant }}{ }^{-1}\right)\end{array}$ & 229.71 & 22.26 & 549.40 & 199.92 & 17.22 & 512.40 & 174.05 & 14.99 & 478.8 \\
\hline 3. & $\begin{array}{l}75 \% \mathrm{~N}-\mathrm{CDU}+(40: 200 \\
\mathrm{g} \mathrm{P}_{2} \mathrm{O}_{5}: \mathrm{K}_{2} \mathrm{O}+10 \mathrm{~kg} \\
\left.\mathrm{FYM} \text { plant }^{-1}\right)\end{array}$ & 221.09 & 22.82 & 548.80 & 190.51 & 18.18 & 520.80 & 167.78 & 15.69 & 484.4 \\
\hline 4. & $\begin{array}{l}50 \% \mathrm{~N}-\mathrm{CDU}+(40: 200 \mathrm{~g} \\
\mathrm{P}_{2} \mathrm{O}_{5}: \mathrm{K}_{2} \mathrm{O}+10 \mathrm{~kg} \mathrm{FYM} \\
\left.\text { plant }^{-1}\right)\end{array}$ & 213.25 & 22.64 & 534.80 & 184.24 & 18.50 & 520.80 & 162.29 & 16.20 & 492.8 \\
\hline 5. & $\begin{array}{l}25 \% \mathrm{~N}-\mathrm{CDU}+(40: 200 \\
\mathrm{g} \mathrm{P}_{2} \mathrm{O}_{5}: \mathrm{K}_{2} \mathrm{O}+10 \mathrm{~kg} \\
\left.\text { FYM }^{-1} \text { plant }^{-1}\right)\end{array}$ & 210.90 & 23.28 & 543.20 & 178.75 & 19.07 & 504.00 & 159.15 & 16.58 & 492.8 \\
\hline 6. & Absolute control & 181.90 & 19.01 & 487.25 & 172.48 & 15.44 & 456.40 & 146.61 & 12.37 & 411.6 \\
\hline & Initial value & 188.16 & 21.43 & 548.8 & 188.16 & 21.43 & 548.8 & 188.16 & 21.43 & 548.8 \\
\hline & S.E. \pm & 1.280 & 0.283 & 10.97 & 1.001 & 0.170 & 5.537 & 1.080 & 0.128 & 4.61 \\
\hline & $\mathrm{CD}$ at $5 \%$ & 3.859 & 0.854 & 33.07 & 3.020 & 0.513 & 16.69 & 3.257 & 0.388 & 13.90 \\
\hline
\end{tabular}

Table.6 Effect of crotonylidenediureaon soil available micronutrient of soil

\begin{tabular}{|c|c|c|c|c|c|c|c|c|c|c|c|c|c|}
\hline \multirow{3}{*}{$\begin{array}{l}\text { Sr. } \\
\text { No }\end{array}$} & \multirow{3}{*}{ Treatment } & \multicolumn{12}{|c|}{ Soilavailablemicronutrient $\left(\mu \mathrm{g} \mathrm{g}^{-1}\right)$} \\
\hline & & \multicolumn{4}{|c|}{ Grand growth stage } & \multicolumn{4}{|c|}{ Shooting stage } & \multicolumn{4}{|c|}{ Harvest stage } \\
\hline & & $\mathbf{F e}$ & Mn & $\mathbf{C u}$ & $\mathbf{Z n}$ & $\mathbf{F e}$ & Mn & $\mathbf{C u}$ & $\mathbf{Z n}$ & $\mathbf{F e}$ & Mn & $\mathbf{C u}$ & $\mathbf{Z n}$ \\
\hline 1. & $\begin{array}{l}\text { GRDN }(200: 40: 200 \mathrm{~g} \\
\mathrm{N}^{\mathrm{P}} \mathrm{P}_{2} \mathrm{O}_{5}: \mathrm{K}_{2} \mathrm{O}+10 \mathrm{~kg} \\
\left.\text { FYM } \text { plant }^{-1}\right)\end{array}$ & 6.32 & 13.82 & 2.98 & 1.08 & 5.35 & 13.03 & 2.33 & 0.81 & 4.34 & 12.13 & 2.49 & 0.70 \\
\hline 2. & $\begin{array}{l}100 \% \mathrm{~N}-\mathrm{CDU}+ \\
\left(40: 200 \mathrm{~g} \mathrm{P}_{2} \mathrm{O}_{5}: \mathrm{K}_{2} \mathrm{O}+\right. \\
\left.10 \mathrm{~kg} \mathrm{FYM} \mathrm{plant}^{-1}\right) \\
\end{array}$ & 6.37 & 13.87 & 2.99 & 1.10 & 5.51 & 13.10 & 2.45 & 0.84 & 4.48 & 12.28 & 3.06 & 0.70 \\
\hline 3. & $\begin{array}{l}75 \% \mathrm{~N}-\mathrm{CDU}+ \\
\left(40: 200 \mathrm{~g} \mathrm{P}_{2} \mathrm{O}_{5}: \mathrm{K}_{2} \mathrm{O}+\right. \\
\left.10 \mathrm{~kg} \mathrm{FYM} \mathrm{plant}^{-1}\right) \\
\end{array}$ & 6.21 & 13.60 & 3.10 & 1.07 & 5.57 & 13.08 & 2.46 & 0.92 & 4.63 & 12.36 & 2.49 & 0.61 \\
\hline 4. & $\begin{array}{l}50 \% \text { N-CDU+ } \\
\left(40: 200 \text { g P }_{2} \mathrm{O}_{5}: \mathrm{K}_{2} \mathrm{O}+\right. \\
\left.10 \mathrm{~kg} \mathrm{FYM} \mathrm{plant}^{-1}\right)\end{array}$ & 6.15 & 13.75 & 3.05 & 1.11 & 5.55 & 13.00 & 2.51 & 0.90 & 4.53 & 12.45 & 2.55 & 0.55 \\
\hline 5. & $\begin{array}{l}25 \% \mathrm{~N}-\mathrm{CDU}+ \\
\left(40: 200 \mathrm{~g} \mathrm{P}_{2} \mathrm{O}_{5}: \mathrm{K}_{2} \mathrm{O}+\right. \\
\left.10 \mathrm{~kg} \mathrm{FYM} \mathrm{plant}^{-1}\right)\end{array}$ & 6.10 & 12.50 & 3.09 & 0.99 & 5.48 & 13.02 & 2.35 & 0.91 & 4.70 & 12.46 & 2.58 & 0.57 \\
\hline 6. & Absolute control & 5.84 & 12.20 & 2.37 & 0.76 & 5.26 & 11.69 & 2.04 & 0.66 & 4.42 & 11.42 & 2.44 & 0.50 \\
\hline & Initial value & 6.27 & 12.78 & 2.92 & 0.96 & 6.27 & 12.78 & 2.92 & 0.96 & 6.27 & 12.78 & 2.92 & 0.96 \\
\hline & S.E. \pm & 0.043 & 0.068 & 0.051 & 0.052 & 0.079 & 0.064 & 0.059 & 0.022 & 0.05 & 0.065 & 0.051 & 0.010 \\
\hline & $\mathrm{CD}$ at $5 \%$ & 0.131 & 0.207 & 0.115 & 0.158 & N.S & 0.193 & 0.180 & 0.066 & 0.17 & 0.196 & 0.155 & 0.031 \\
\hline
\end{tabular}


Table.7 Effect of crotonylidenediurea on Biological properties of soil

\begin{tabular}{|c|c|c|c|c|c|c|c|c|c|c|}
\hline \multirow[t]{2}{*}{$\begin{array}{l}\text { Sr. } \\
\text { No }\end{array}$} & \multirow[t]{2}{*}{ Treatment } & \multicolumn{3}{|c|}{$\begin{array}{c}\text { Urease enzyme } \\
\left(\text { ug } \mathrm{NH}_{4}-\mathrm{N} \mathrm{g}^{-1} \text { of soil } \mathrm{hr}^{-1}\right)\end{array}$} & \multicolumn{3}{|c|}{$\begin{array}{c}\text { DHA enzyme } \\
\left(\text { ug TPF } \mathrm{g}^{-1} \text { soil hr }^{-1}\right)\end{array}$} & \multicolumn{3}{|c|}{$\begin{array}{l}\text { Bacterial count } \\
(\text { CFU x 10 }\end{array}$} \\
\hline & & $\begin{array}{l}\text { Grand } \\
\text { growth }\end{array}$ & $\begin{array}{l}\text { Shooting } \\
\text { Stage }\end{array}$ & $\begin{array}{c}\text { Harvest } \\
\text { stage }\end{array}$ & $\begin{array}{l}\text { Grand } \\
\text { growth }\end{array}$ & $\begin{array}{c}\text { Shooting } \\
\text { stage }\end{array}$ & $\begin{array}{c}\text { Harvest } \\
\text { stage }\end{array}$ & $\begin{array}{l}\text { Grand } \\
\text { growth }\end{array}$ & $\begin{array}{l}\text { Shooting } \\
\text { stage }\end{array}$ & $\begin{array}{c}\text { Harvest } \\
\text { stage }\end{array}$ \\
\hline 1. & $\begin{array}{l}\text { GRDN }(200: 40: 200 \mathrm{~g} \\
\mathrm{N}: \mathrm{P}_{2} \mathrm{O}_{5}: \mathrm{K}_{2} \mathrm{O}+10 \mathrm{~kg} \\
\text { FYM plant } \\
\end{array}$ & 30.80 & 34.65 & 32.38 & 1.35 & 1.44 & 1.42 & 21.00 & 22.75 & 21.50 \\
\hline 2. & $\begin{array}{l}100 \% \mathrm{~N}-\mathrm{CDU}+ \\
\left(40: 200 \mathrm{~g} \mathrm{P}_{2} \mathrm{O}_{5}: \mathrm{K}_{2} \mathrm{O}+\right. \\
\left.10 \mathrm{~kg} \mathrm{FYM} \mathrm{plant}^{-1}\right)\end{array}$ & 28.00 & 31.50 & 29.58 & 1.24 & 1.32 & 1.29 & 20.50 & 22.50 & 21.50 \\
\hline 3. & $\begin{array}{l}75 \% \mathrm{~N}-\mathrm{CDU}+(40: 200 \\
\mathrm{g}_{2} \mathrm{O}_{5}: \mathrm{K}_{2} \mathrm{O}+10 \mathrm{~kg} \\
\left.\mathrm{FYM} \mathrm{plant}^{-1}\right)\end{array}$ & 25.90 & 28.88 & 27.46 & 1.18 & 1.24 & 1.21 & 20.00 & 21.50 & 20.25 \\
\hline 4. & $\begin{array}{l}50 \% \mathrm{~N}-\mathrm{CDU}+(40: 200 \\
\mathrm{g} \mathrm{P}_{2} \mathrm{O}_{5}: \mathrm{K}_{2} \mathrm{O}+10 \mathrm{~kg} \\
\left.\mathrm{FYM} \mathrm{plant}^{-1}\right)\end{array}$ & 24.68 & 27.65 & 26.08 & 1.17 & 1.20 & 1.19 & 19.00 & 21.00 & 19.00 \\
\hline 5. & $\begin{array}{l}25 \% \mathrm{~N}-\mathrm{CDU}+(40: 200 \\
\mathrm{g} \mathrm{P}_{2} \mathrm{O}_{5}: \mathrm{K}_{2} \mathrm{O}+10 \mathrm{~kg} \\
\left.\text { FYM }^{-1} \text { plant }^{-1}\right)\end{array}$ & 23.10 & 27.30 & 24.50 & 1.06 & 1.10 & 1.08 & 18.00 & 19.75 & 18.25 \\
\hline \multirow[t]{4}{*}{6.} & Absolute control & 19.95 & 20.30 & 20.30 & 0.98 & 1.01 & 1.00 & 17.75 & 18.25 & 16.75 \\
\hline & Initial value & \multicolumn{3}{|c|}{19.60} & \multicolumn{3}{|c|}{0.97} & \multicolumn{3}{|c|}{17.25} \\
\hline & S.E. \pm & 0.220 & 0.445 & 0.334 & 0.017 & 0.013 & 0.018 & 0.562 & 0.430 & 0.430 \\
\hline & $\mathrm{CD}$ at $5 \%$ & 0.664 & 1.342 & 1.007 & 0.051 & 0.041 & 0.055 & 1.694 & 1.297 & 1.794 \\
\hline
\end{tabular}

It was decreased with decreased levels of nitrogen application through CDU. The reducing sugars content of banana was found statistically on par with each other in GRDN, 100, 75 and $50 \% \mathrm{~N}$ through $\mathrm{CDU}(15.04$, $14.79,14.70$ and 14.66 per cent respectively). Significantly lower reducing, non reducing and total sugars of 14.16, 1.48 and $15.71 \%$ were recorded by the treatment of absolute control. Similar finding have also been reported by Ram (1999) and Kavino et al., (2003). The pulp:peel ratio was increased numerically with increase in the level of nitrogen for banana. Application of GRDN recorded significantly higher pulp:peel ratio of 3.60 (Table 4 ) over the rest of treatment.

The residual soil fertility at harvest of banana was significantly influenced by the GRDN and nitrogen application to banana through CDU. The soil available nitrogen content was significantly more in GRDN (178.75 kg ha-1) and decrease in treatment 100, 75, 50 and 25
$\% \mathrm{~N}$ through CDU (Table 5). The residual soil available phosphorus was found statistically on par with each other in all the treatment except absolute control but less than the initial soil available phosphorus content (21.43 kg ha-1). The soil available potassium content in GRDN, 100, 75, 50 and $25 \% \mathrm{~N}$ through CDU was found statistically on par with each other (478.8, 478.8, 484.4, 492.8 and $492.8 \mathrm{~kg}$ ha-1 respectively) but considerably less than initial soil available potassium content $(548.80 \mathrm{~kg}$ ha- 1$)$.

The soil available micronutrients viz., Fe, Mn, $\mathrm{Cu}$ and $\mathrm{Zn}$ was significantly influenced by GRDN and nitrogen application through CDU (Table 6). The manganese (12.13 and 12.28 $\mu \mathrm{g}$ g-1 soil) and zinc (0.70 and $0.70 \mu \mathrm{g}$ g- 1 soil) content was statistically on par with GRDN and $100 \% \mathrm{~N}$ through CDU, whereas, copper content was significantly higher in 100 $\% \mathrm{~N}$ through CDU (3.06 $\mu \mathrm{g} \mathrm{g}-1$ soil). The urease and dehydrogenase soil enzyme 
activity was significantly higher are presented in general recommended dose of nutrients (34.65 $\mu \mathrm{gNH} \neg 4+-\mathrm{N}$ g-1 of soil $\mathrm{hr}-1$ and 1.44 $\mu \mathrm{g}$ TPF g-1 soil hr-1)(Table 7). It was followed by $100 \% \mathrm{~N}$ through CDU(31.50 $\mu \mathrm{g}$ NH $\neg 4+-\mathrm{N}$ g-1 of soil hr-1 and $1.32 \mu \mathrm{g}$ TPF g1 soil hr-1).The bacterial population count in treatment GRDN, $100 \%$ and $75 \% \mathrm{~N}$ through CDU were statistically on par with each other (21.50, 21.50 and $20.25 \mathrm{cfu} x 106 \mathrm{~g}-1$ soil respectively). Maximum bacterial population observed in grand growth stage in GRDN and $100 \% \mathrm{~N}$ through CDU and thereafter bacterial population was reduced up to harvest (Table 6). These results are in close conformity with the results of Xiaoguang et al., (2004), Chang et al., (2006) and Goutami et al., (2015).

\section{References}

Allen, S.E. 1986. Slow release nitrogen fertilizers in crop production. ASA., CSSA, Madison, 192-206.

Alvan, A.K. and Tucker, D.P. 1996. Evaluation of a resin coated nitrogen fertilizer for young citrus trees on a deep sand. Proc. Fla. State Horticulture Society, 106, 4-8.

Aquil, B., Jan, A.T., Sarin, N.B. and Haq, Q.R. 2012. Micro propagation and genetic transformation of banana for crop improvement and sustainable agriculture. J. Crop Sci., 3: 64-77.

Babu, N. and Sharma, A. 2005. Effect of integrated nutrient management on productivity of 'Jahajee' banana and soil properties under Nagaland foot hills conditions. Orrisa J. Horticulture, 33: 3134.

Badgujar, C.D., Dusane, S.M. and Deshmukh, S.S. 2004. Influence of plant spacing on growth, maturity and yield of Grand Naine (AAA). banana. South Indian Horticulture, 52: 13-17.

Brady, N.C and Weil, R.P. 1999. Soil organic matter. In The nature and properties of soils, (Brady, N.C, Weil, R.P., Eds.),
Upper Saddle river, New Jersey, pp. 446490

Casida, L.E., Klein, D.A. and Sautor, T. 1964. Soil dehydrogenase activity. Soil Sci., 98: 371-376.

Chapman, H.D. and Pratt, P.F. 1961. Methods of Analysis for Soil, Plant and Water.Division of Agricultural Sciences, California University USA.

FAO. 2015. Food and Agriculture Organization of the united Nations.

Follett, R.F. 2001. Innovative 15N microplot research techniques to study nitrogen use efficiency under different ecosystems. In (Mills, H. A, eds.), Communications in Soil Science and Plant Analysis, 32: 951979.

Frank, T. and Malkomes, H.P. 1993. Influence of tem $\neg$ perature on microbial activities and their reaction to the herbicide "Goltix" in different soils under laboratory conditions. Zentralblatt fur Mikrobiologie, 148:403-412.

Gour, G.R., Tomar, S.S., Rathi, G.S. and Tomar, G.S. 1990. Studies on the relative efficiency of slow release nitrogenous fertilizers and nitrification inhibitors in lowland rice. Narendra Deva J. Agri. Res., 5: 26-34.

Goutami, N., Rani, P.P., Pathy, R.L and Babu, P.R. 2015. Soil properties and biological activity as influenced by nutrient management in Rice-fallow Sorghum. Int. J. Agri. Res. Innovations and Technol., 5: 10-14.

Jackson, M.L. 1973. Soil Chemical Analysis, Prentice Hall of India Pvt. Ltd., New Delhi.

Kavino, M., Kumar, N., Soorianathasundaram, K. and Jeykumar, P. 2003. Effect of fertigation on the growth and development of first ratoon crop (R-1. of banana cv. Robusta (AAA. under high density planting system. Indian $J$. Horticulture, 61: 39-41.

Lahav, E. 1995. Banana nutrition. In (Gowen, S. ed.),Bananas and plantains. Chap $\neg$ man and Hall, New York, pp. 258-316. 
Lane, J.H. and Eynon, L. 1923. Determination of sugars by fehling solution methylene blue as indicator. J. Chem. Society of India, 42: 32-34.

Lindsay, W.L. and Norvell, W.A. 1978. Development of DTPA soil test for zinc, iron, manganese, and copper. Soil Sci. Society of America J., 42: 421-428.

Lorenz, O.A. 1978. Potential nitrate levels in edible plant parts. In, (Nielsen, D.R. and J.G. MacDonald eds.), Nitrogen in the environment, Vol. 2. Soil plant nitrogen relationships. Academic Press, New York, San Francisco, London, pp. 201252.

Mansour, A.E., Ahmed F.F., Abdelaal, A.M and Cimpoies, G.P. 2007. Use of mineral, organic, slow release and biofertilizers for Anna apple trees in a sandy soil. African Crop Science Conference Proceedings, 8: 265-271.

Mustaffa, M.M. 1988. Effect of spacing and nitrogen on growth, fruit and yield of Robust banana grown under rainfed conditions. South Indian Horticulture, 36: 228-231.

Perucci P., Scarponi, L. and Businelli, M. 1984. Enzyme activities in a clay-loam soil amended with various crop residues. Plant and Soil, 81: 345-351.

Ram, R.A. 1999. Effect of controlled-release fertilizers on growth, yield and fruit quality of guava cv. Sardar in Ustochrepts. Indian J. Horticulture, 56, 104-111.
Reddy, G.B. and Faza, A. 1989. Dehydrogenase activity in sludge amended soil. Soil Biol. Biochem., 21: 327.

Shaviv, A. and Mikkelsen, R.L. 1993. Controlled-release fertilizer to increase efficiency of nutrient use and minimize environmental degradation. A Review Fertilizer Res., 35: 1-12.

Srinivas, K., Reddy, B.M.C., Chandra, S.S., Thimooe Gowda., Raghupati, H.B. and Padma, P. 2001. Growth, yield and nutrient uptake of Robust banana in relation to $\mathrm{N}$ and $\mathrm{K}$ fertigation. Indian $J$. Horticulture, 58: 287-293.

Susherman, S. and Anggoro. 2011. Producing slow release urea by coating Starch/Acrylic in fluid Bed Sprayinging. IJET-IJENS, 11: 77-80.

Tabatabai, M.A. and Bremner, J.M. 1972. Assay of urease activity in Soil. Soil Biol. Biochem., 4: 479-487.

Wilke, B.M. 1991. Effects of single and successive ad $\neg$ ditions of cadmium, nickel and zinc on carbon dioxide evolution and dehydrogenase activity in a sandy Luvisol. Biol. Fertility of Soils, 11: 34-37.

Xiaoguangjiao, Liang wenju., Chen lijun, Zhang haijun and Li qi wangpeng. 2004. Effects of slow-release urea fertilizers on urease activity, microbial biomass, and nematode communities in an aquatic brown soil. Sci. China Life Sci., 48: 2632.

\section{How to cite this article:}

Atul Pawar, M.R. Chauhan and Pramod Gawade. 2017. Effect of Crotonylidene Di-Urea on Yield and Quality of Banana on Inceptisol. Int.J.Curr.Microbiol.App.Sci. 6(5): 2171-2180.

doi: https://doi.org/10.20546/ijcmas.2017.605.244 